\title{
IN VITRO TESTING OF AFRICAN TRADITIONAL MEDICINES FOR CYTOTOXIC, IMMUNE MODULATORY AND ANTI-HIV ACTIVITIES
}

\author{
Nceba Gqaleni ${ }^{a}$, Mlungisi Ngcobo ${ }^{a}$, Raveen Parboosing ${ }^{\mathrm{b}}$, and Anneta Naidoo ${ }^{\mathrm{b}}$ \\ ${ }^{a}$ Traditional Medicine Laboratory, ${ }^{b}$ Department of Virology/National Health Laboratory Service (NHLS), \\ Nelson R Mandela School of Medicine, University of KwaZulu-Natal Durban, South Africa. \\ * E-mail: gqalenin@ukzn.ac.za
}

\begin{abstract}
African Traditional Medicines (ATMs) serve as a major source of primary healthcare for African people. The reasons for their use range from easy access, affordability, beliefs in traditional systems and long term safety. ATMs have been used to treat individuals infected with HIV and therefore need scientific validation; a view supported by Traditional Health Practitioners (THPs). This study aimed to evaluate the in vitro cytotoxicity, immune modulatory and anti-HIV activities of traditional multiple herbal preparations from local THPs. Ugambu, Ihashi, Product Nene, Product Blue, SPNa and SDKc ATM were supplied by local THPs. Changes in adenosine triphosphate (ATP) \& glutathione (GSH) over 24 hours were measured using luminometry. Changes in 12 cytokines were assayed using an ELISA-based absorbance assay. Protective effects against HIV killing of MT-4 cells were tested using the XTT assay and antiviral activity was measured using an HIV-1 viral load assay. Cyclosporine and AZT were used as positive controls. Ugambu, Ihashi, Product Nene and SDKc induced a dose dependent toxicity on treated PBMCs by reducing ATP and GSH at high doses $(p<0.001)$. These medicinal preparations, along with SPNa, showed immunomodulatory activity by significantly $(p<0.001)$ changing the secretion of pro-inflammatory cytokines. Product Blue stimulated the levels of ATP and GSH in treated PBMCs at all doses however this product did not show any immunomodulatory activity on cytokine secretion when compared to control cells. Ugambu, Ihashi, Product Nene showed promising anti-HIV activity relative to AZT $(p<0.01)$. This study has shown that some of these traditional medicinal preparations have at least one or all the properties of immunostimulation, immunomodulation or antiretroviral effects. The mechanism of action of the shown activities should further be investigated.
\end{abstract}

Keywords: African traditional medicines, HIV/AIDS, cytotoxicity, immunomodulation, antivirals

\section{Introduction}

Traditional health practitioners (THPs) serve as primary healthcare providers for a large population of African people. Traditional medicines from these THPs are preferred by African people because of their perceived safety, long history of their use and because they are easily accessible, among other reasons. The use of these traditional or herbal medicines in the African continent is largely based on oral tradition within the family or community (WHO, 2005; Tshibangu et al., 2004). Worldwide, the trend has also shifted towards the use of traditional medicines due to concerns about the costs, invasiveness and potential for toxicity of conventionally mainstream remedies (Cowan, 1999).

With the spread of HIV and AIDS, there has been an increase in traditional medicine preparations which purport to have immune "boosting" or modulatory activity. The approach of African traditional medicines (ATM) to fighting infections has the concept of "boosting the immune system" as one of its central pillars. Therefore it is important to study the effects of ATM on the immune response. Regarding herbal medicines, immunomodulators may be defined as herbal medicinal extracts that modify the immune response via regulation of cytokines, hormones, neurotransmitters, and other peptides. Many herbal medicinal extracts have immune modulatory activity on at least one cytokine (Spelman et al., 2006).

The immune system is increasingly playing a role in progression of chronic diseases for which conventional drugs have limited ability to treat and especially prevent. Therefore it appears sensible to target the immune response in order to alter the threat of certain chronic diseases (Haddad et al., 2005). Strengthening of the immune response is very important in debilitating conditions such as cancer and AIDS. The immune system is responsible for the complex task of providing defense against a vast array of potential pathogens, whilst ensuring that the same protective mechanisms are not turned against self (Lowe and Lin, 2000). The rise in antibiotic resistance and the decline in the development of new classes of conventional antibiotics have necessitated development of new strategies in the treatment of microbial infections. Because the primary target of an immunomodulatory treatment is the host and the effects on the pathogen are exerted indirectly via augmentation of the immune response, the selective pressure for pathogen resistance to the drug is minimized (Nijnik et al., 2010).

Most of the studied herbal medicinal extracts are made of a single plant while most of the traditional medicines are made up of two or more plants mixed together. Although multiple herbal mixtures are extensively studied in the Far East these approaches are only beginning to become available to Western scientists. Most African traditional medicines follow the same 
concept of multiple herbal mixtures. With an increase in the popularity and attractiveness in Western society of complementary and alternative medicine (CAM), there is increasing interest within the scientific community in studying the safety, efficacy and mechanism of multiple herbal medicines (Chen et al., 2002). Studies on traditional multiple herbal preparations are also becoming of interest to South African scientists (Bessong et al., 2004; Tshibangu et al., 2004; Ndhlala et al., 2009; Ndhlala et al., 2010). We therefore aimed to study the in vitro cytotoxicity, immune modulatory and anti-HIV activities of traditional multiple herbal preparations from local THPs.

\section{Materials and Methods \\ Reagents and equipment}

RPMI-1640 with HEPES buffer (25mM), foetal calf serum (FCS), penstrep-fungizone (PSF), and L-glutamine were purchased from Adcock Ingram (SA). Normal whole blood was obtained from the South African National Blood Supply (SANBS). Histopaque 1077, 2,3-bis[2-Methoxy-4-nitro-5-sulfophenyl]-2H-tetrazolium-5-carboxyanilide (XTT) kit and cyclosporine were purchased from Sigma Aldrich (USA). Ethanol was bought from Merck Chemicals (SA). Promega CellTiterGlo $^{\mathrm{TM}}$ Luminescent Cell Viability was purchased from Promega ${ }^{\mathrm{TM}}$ (USA). The Multi-Analyte Profiler ELISArray ${ }^{\mathrm{TM}}$ Kit was purchased from SABiosciences (USA). Modulus ${ }^{\mathrm{TM}}$ Microplate Luminometer was from Turner BioSystems (USA). The 96-well plate reader was acquired from Bio-Tek (SA), NucliSens HIV-1 EasyQ assay kit was purchased from BioMerieux, Marcy I'Etoile (France).

\section{Preparation of medicinal plant extracts}

The herbal preparations were obtained from local THPs who consented to their products being part of this research. The participation of THPs was protected under a material transfer agreement (MTA) signed between each THP and the University of KwaZulu Natal (UKZN). Some of the medicinal preparations are commercially available while others are prescribed by THPs during consultations with their patients. For the purpose of this paper the results of the following products will be presented: Ugambu, Ihashi, Product Nene, Product Blue (Spray), SPNa and SDKc. The medicinal preparations were requested to be freshly prepared from THPs for in vitro assays. Those supplied in liquid form were autoclaved and filter-sterilised under vacuum twice through $0.22 \mu \mathrm{m}$ filters for tissue culture assays. The samples were preserved by freeze drying and stored at $-20{ }^{\circ} \mathrm{C}$ for later use. Medicinal preparations supplied by THPs in unextracted plant material form were extracted according the healers instructions (boiled in distilled water), cooled over night and then autoclaved, filter-sterilised under vacuum and preserved as described above. The prepared extracts were then diluted into different treatment doses using cell culture media (CCM). The dilutions were prepared on the day the cells were treated.

\section{Isolation of peripheral blood mononuclear cells (PBMCs) from whole blood}

Normal whole blood from healthy volunteers acquired (from the SANBS research in EDTA coated containers) after prior informed consent was obtained in line with the South African Ethical Advisory Board was carefully layered onto equal amounts of Histopaque 1077 then centrifuged at $400 \mathrm{~g}$ for 30 minutes at $25{ }^{\circ} \mathrm{C}$. After centrifugation, the buffy coat layer, containing PBMCs, was isolated and washed twice in phosphate buffered-saline (PBS, $5 \mathrm{ml}),\left(1150 \mathrm{~g}\right.$ for 20 minutes at $\left.25^{\circ} \mathrm{C}\right)$. The isolated PBMCs were diluted in RPMI-1640 and pooled into one tube. A cell count of $1.5-2 \times 10^{6} \mathrm{cells} / \mathrm{ml}$ was determined using the trypan blue method to standardise the cell dilution.

\section{Treatment of PBMCs with medicinal preparations}

Aliquots of the cell suspension $(2 \mathrm{ml})$ were transferred into each well in a 6-multiwell plate. Dilutions of the medicinal extracts $(2 \mathrm{ml})$ in cell culture media were then added to each of the treatment wells. Different doses of cyclosporine were used as positive controls and untreated control samples were also included as reference samples. The 6-well plates were then incubated for 24 hours at $37^{\circ} \mathrm{C}$ in a $5 \% \mathrm{CO}_{2}$ incubator.

\section{Cell viability assay}

Following the incubation of treated cells for $24 / 48$ hours, a $100 \mu 1$ of cells from each treatment concentration was pipetted into three different wells of a white opaque 96-well plate. The CellTiter-Glo Reagent (Promega, cat number: G7570) was mixed immediately before use and was added to the wells with treated cells at $100 \mu \mathrm{l}$ per well. The plate was shaken on a plate shaker for 2 minutes at $30 \mathrm{~g}$. This plate was then incubated in the dark for 10 minutes at room temperature and the relative light units (RLU) of the samples were measured in a luminometer following the manufacturer's instructions. A dose response curve was also generated for the ATP levels using the RLU and the dilutions of medicinal preparations and different control samples. 
Intracellular glutathione assay

Changes in intracellular content of treated and control samples were measured using a luminometry based GSH-Glo assay kit from Promega (cat number: V6911). Following the incubation of treated cells for 24 hours, the cell culture media was removed from experimental samples. These samples were washed twice with PBS and a $100 \mu \mathrm{l}$ of cells from each treatment concentration was pipetted into three different wells of a white opaque 96-well plate. The GSH-Glo reagent for suspension cultures was prepared by diluting the Luciferin-NT substrate and Glutathione-S-Transferase 1:50 in GSH-Glo reaction buffer. Each reaction (well) of a 96-well plate required $50 \mu \mathrm{l}$ of the GSH-Glo reagent and the plate was then incubated in the dark for 30 minutes at room temperature. The luciferin detection reagent was prepared by transferring the contents of one bottle of luciferin detection buffer to the bottle of lyophilised luciferin detection reagent. The contents were mixed by inversion several times until the substrate was thoroughly dissolved. Each reaction (well) of a 96-well plate required $100 \mu 1$ of the luciferin detection reagent meaning the luciferin detection reagent needed to be 2:1 with the GSH-Glo reagent and this incubated for 15 minutes in the dark at room temperature. At the end of the two step reaction the plate was read using a luminometer by following manufacturer's instructions.

\section{Cytokine expression assay}

The Multi-Analyte Profiler ELISArray assay kit (SA Biosciences, cat number: MEH-004A) is designed to be used with supernatants from treated cells or with serum from whole blood. It is a variation of the normal ELISA kit which uses absorbance instead of fluorescence to read the final results. The kit also does not give the exact concentration of each cytokine in pico or nanograms as a normal ELISA kit but differences in absorbance readings between controls and treated samples gives changes in cytokine secretion. For the purpose of this research, cytokines were analysed from supernatants of treated PBMCs. Each kit included 96-well plate coated with antibodies for the various cytokines in the microarray. Each row of the plate from 1 to 12 represented a single cytokines in the following order: IL $1 \alpha$, IL 1 $\beta$, IL 2, IL 4, IL 6, IL 8, IL 10, IL 12, IL 17 $\alpha$, IFN- $\gamma$, TNF- $\alpha$, and GM-CSF. The kit had negative and positive method controls. Each sample was assayed in duplicate. Briefly, incubation of the samples in the 96-well plates allowed the capture antibodies to bind their specific protein of interest. After washing away unbound protein with wash buffer, biotinylated detection antibodies $(50 \mu \mathrm{l})$ were added to the wells to also bind the captured analyte. Following another wash, an avidin-horseradish peroxidase conjugate $(100 \mu l)$ was added. The wells were again washed and the colorimetric substrate solution was added, developing to a blue colour in direct proportion to the amount of protein analyte present in the initial sample. The colour development was stopped by adding the stop solution, and the absorbance was read at 450 $\mathrm{nm}$ with reference at $570 \mathrm{~nm}$ in a microplate reader as per manufacturer's instructions.

\section{2,3-bis[2-Methoxy-4-nitro-5-sulfophenyl]-2H-tetrazolium-5-carboxyanilide (XTT) Screening Assay}

The cytotoxic and potential antiviral effects of the plant extracts were determined using the In Vitro Toxicology assay kit XTT based (Sigma-Aldrich, Missouri, USA) (Scudiero et al., 1988; Weislow et al., 1989; Roehm et al., 1991). Briefly, MT-4 cells were seeded into the wells of 96-well culture plates at $6 \times 10^{5}$ cells $/ \mathrm{ml}$. The virus stain HTLV $\mathrm{V}_{\text {IIIB }}$ was used to infect MT-4 cells at 100-300 TCID $_{50}$. Five-fold dilutions of the plant extracts were added to the plate. The plate was incubated for 5 days at $37^{\circ} \mathrm{C}$ in a $\mathrm{CO}_{2}$ incubator. After 5 days, the XTT salt was added to the wells and incubated for 4 hours at $37^{\circ} \mathrm{C}$ in a $\mathrm{CO}_{2}$ incubator to determine the viability of the cells. The absorbances were read at $450 \mathrm{~nm}$ (reference $620 \mathrm{~nm}$ ). The Inhibitory Concentration at $50 \%\left(\mathrm{IC}_{50}\right)$, Effective Concentration at 50\% $\left(\mathrm{EC}_{50}\right)$ and Selectivity Index $(\mathrm{SI})$ were calculated for each substance.

\section{HIV-1 Viral load assay}

MT-4 cells were seeded into 6 well plates at $1 \times 10^{6}$ cells $/ \mathrm{ml}$. Ten-fold dilutions were performed on the plant extracts and added to the plates. The cells and extracts were incubated for 30 minutes to achieve drug/cell equilibrium. HTLV $V_{\text {IIIB }}$ was added at $100 \mathrm{TCID}_{50}$ to the plates and incubated for 3 hours. Cells were washed twice with PBS and centrifuged at $350 \mathrm{x}$ g for 5 minutes. The cells were resuspended in RPMI-1640 containing the plant extracts at varying concentrations. The plates were incubated at $37^{\circ} \mathrm{C}$ in $\mathrm{CO}_{2}$ for 5 days. Supernatant for viral load testing was removed from each well at days $0,1,3$ and 5 . The NucliSens HIV-1 EasyQ assay (BioMerieux, Marcy I'Etoile, France) was used to quantify the virus. The results were represented graphically and compared to the known antiviral activity of AZT.

\section{Statistical analyses}

Data analysis was done on Microsoft Excel, Microsoft ${ }^{\mathrm{TM}}$ Corporation, USA, to obtain descriptive statistics. The different levels of significances within the separate treated groups were analysed using one-way analysis of variance (ANOVA) and the differences between the treated cells and the control cells were analysed in GraphPad Instat software (version 3) using the Tukey-Kramer multiple comparison test. Differences with $p<0.05$ were considered statistically significant. 


\section{RESULTS \\ Cell viability assay}

Cell viability results are illustrated in Figure 1 (A-G). Cyclosporine, a positive control, induced a decrease in cell viability with increase in treatment dose (Figure 1A). High doses of Ugambu (Figure 1B) significantly $(p<0.001)$ decreased cell viability while high dilutions were not cytotoxic to treated leukocytes when compared to untreated cells. All doses of Product Blue did not show any cytotoxicity but seemed to stimulate viability of immune cells $(p<0.001)$ (Figure $1 \mathrm{C})$.

Ihashi was shown to be highly cytotoxic to immune cells at high doses up to a dilution of 1/200 $(p<0.001)$. Low doses of this product were tolerated by treated PBMCs when compared to the untreated control (Figure 1D). Product Nene and SDKc also demonstrated dose dependent effects on treated immune cells (Figures 1E and G). Both products were significantly ( $p<$ 0.001) cytotoxic to PBMCs at high doses when compared to untreated cells but Product Nene demonstrated higher cytotoxic than $S D K c$. SPNa doses significantly reduced cell viability even at medium doses (1/300) (Figure 1F).

\section{Intracellular glutathione assay}

Changes in intracellular GSH levels reflect the number of viable cells in a given sample. Therefore the GSH assay also served as a confirmatory assay for the ATP cell viability assay. Cyclosporine was also used as a positive control for this assay and showed a similar dose dependent reduction in GSH levels as was the case with cell viability (Figure 2A). Ugambu had a slightly significant $(p<0.01)$ effect on GSH content at higher doses while low doses increased intracellular GSH (Figure 2B). Product Blue stimulated intracellular levels of GSH in all treatment

Changes in the levels of GSH of PBMCs treated with Ihashi and Product Nene tracked changes in cell viability as measured by the ATP assay (Figure 1D \& E). Both traditional medicine preparations significantly $(p<0.001)$ reduced GSH levels at high doses while low doses were tolerated by treated immune cells when compared to untreated control cells (Figure 2D \& E). SPNa and SDKC did not deplete intracellular GSH levels of PBMCs in all treatment doses when compared to untreated immune cells.

\section{Cytokines assay}

The use of the Multi-Analyte Profiler ELISArray assay kit allowed batch screening of the effects of traditional medicine preparations on pro-inflammatory cytokine secretion in PBMCs in an in vitro model. Changes in absorbance values of supernatants from treated PBMCs were compared to untreated control supernatants. Product Blue was the only product that did not show any significant changes in secretion of all 12 cytokines when compared to untreated samples. Ugambu, Ihashi, Product Nene, SPNa, and SDKc induced significant $(p<0.001)$ changes in pro-inflammatory cytokine secretion at very low treatment doses (Tables 1 and 2). Almost all these changes reflected an increase in cytokine secretion after treatment with dilutions of these traditional medicine preparations.

Table 1: Results of the inflammatory cytokines assay from PBMCs supernatants of treated and untreated samples. Human PBMCs were treated with dilutions of Ugambu, Product Blue and Ihashi over 24 hours. Ugambu and Ihashi showed an ability to promote pro-inflammatory cytokine secretion at low doses while Product Blue did not show any significant change in most of the cytokines analysed.

\begin{tabular}{|c|c|c|c|c|c|c|c|c|c|}
\hline & \multicolumn{4}{|c|}{ Ugambu } & \multicolumn{4}{c|}{ Product Blue } & \multicolumn{4}{c|}{ Ihashi } \\
\cline { 2 - 11 } Cytokines & \multicolumn{9}{|c|}{ Spectrophotometric absorbance readings } \\
\cline { 2 - 11 } & Control & $\mathbf{1 / 2 0 0}$ & $\mathbf{1 / 5 0 0}$ & Control & $\mathbf{1 / 2 0 0}$ & $\mathbf{1 / 5 0 0}$ & Control & $\mathbf{1 / 2 0 0}$ & $\mathbf{1 / 5 0 0}$ \\
\hline IL 1 $\alpha$ & 0.021286 & $0.125202^{*}$ & $0.155075^{*}$ & 0.021286 & 0.040323 & 0.019013 & 0.033957 & 0.019188 & 0.037379 \\
\hline IL 1 $\beta$ & 0.056427 & $0.67238^{*}$ & $0.673929^{*}$ & 0.056427 & 0.071787 & 0.033606 & 0.016937 & 0.026369 & $0.054548^{*}$ \\
\hline IL 2 & 0 & 0 & 0 & 0 & 0.002887 & 0 & 0 & 0 & $0.010967^{*}$ \\
\hline IL 4 & 0.0055906 & $0.035448^{*}$ & $0.043077^{*}$ & 0.005906 & 0.021286 & 0.018566 & 0.022376 & 0.015249 & 0.030553 \\
\hline IL 6 & 2.291123 & 2.317117 & 2.328777 & 2.291123 & 2.292034 & 2.276336 & 0.313504 & 0.4006061 & $1.046472^{*}$ \\
\hline IL 8 & 2.247801 & 2.252264 & 2.283699 & 2.247801 & 2.257167 & 2.275678 & 2.234766 & 2.195429 & 2.241874 \\
\hline IL 10 & 0.140699 & $0.863191^{*}$ & $0.87614^{*}$ & 0.140699 & 0.17455 & 0.101955 & 0.040148 & 0.04316 & $0.071133^{*}$ \\
\hline IL 12 & 0.005759 & $0.014285^{*}$ & $0.041226^{*}$ & 0.005759 & 0.024374 & 0 & 0.023157 & 0.035197 & 0.017104 \\
\hline IL 17 $\alpha$ & 0.009282 & 0.005762 & $0.018934^{*}$ & 0.009282 & 0.027508 & 0 & 0.040553 & 0.032653 & $0.019679^{*}$ \\
\hline IFN $\gamma$ & 0 & $0.021785^{*}$ & $0.0406^{*}$ & 0 & 0.004881 & 0 & 0.029434 & 0.035976 & 0.018466 \\
\hline TNF $\alpha$ & 0.158224 & $0.324455^{*}$ & $0.397102^{*}$ & 0.158224 & 0.054626 & 0.051161 & 0.01857 & $0.034654^{*}$ & 0.016679 \\
\hline GM-CSF & 0.011239 & 0.09674 & $0.124461^{*}$ & 0.011239 & 0.022104 & 0.025594 & 0.009118 & $0.049298^{*}$ & 0.017863 \\
\hline
\end{tabular}

*Significant change in absorbance readings for each cytokine as compared to untreated control sample 


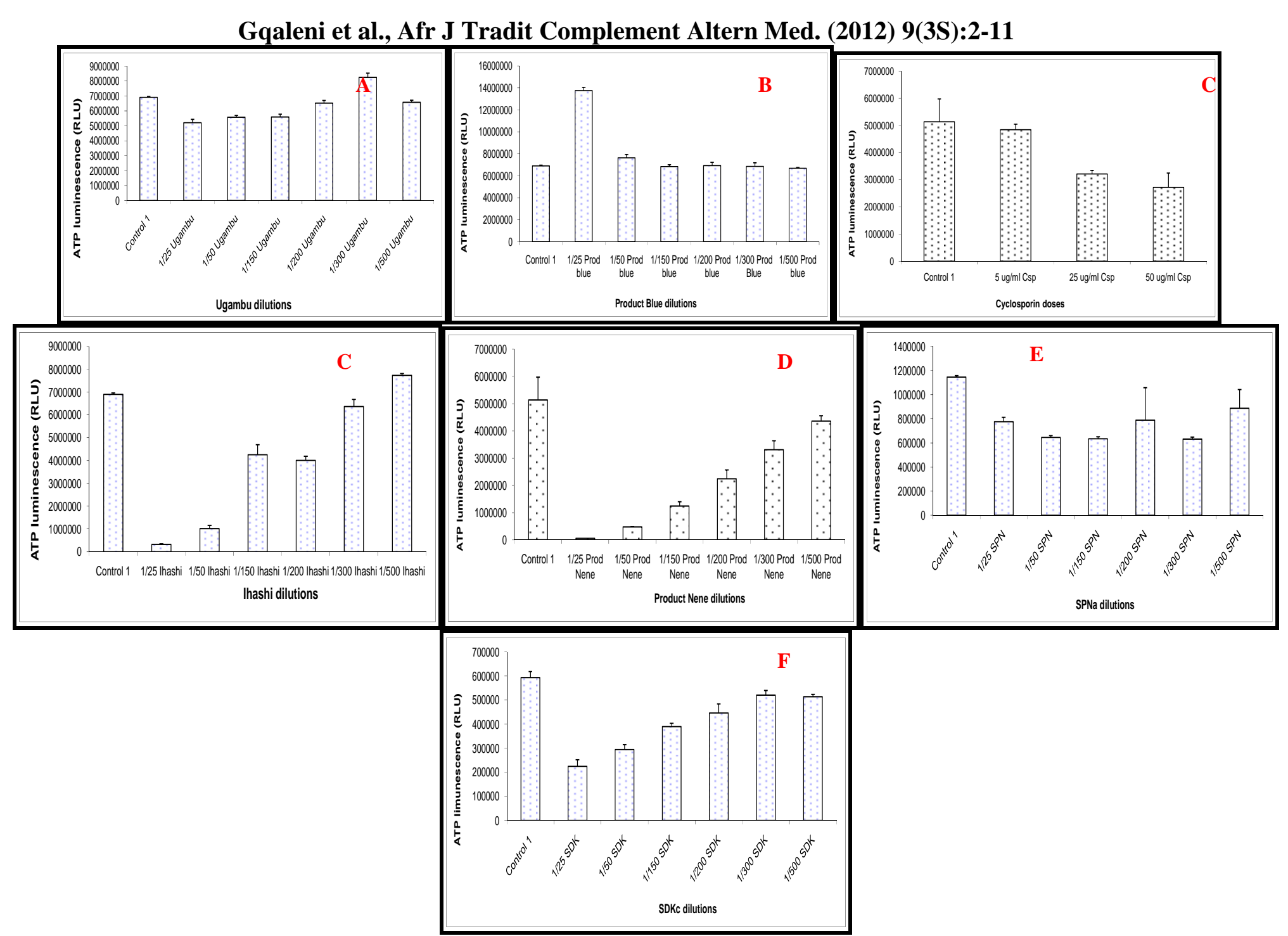

Figure 1: Graphical representation of the effects of traditional medicine preparations on ATP levels, which reflect cell viability, in PBMCs treated with different doses after 24 hours. The dose response curves represented include (A) cyclosporine, (B) Ugambu, (C) Product Blue, (D) Ihashi, (E) Product Nene, (F) SPNa, and (G) SDKc. Each graph includes an untreated control as a reference sample. Cyclosporine (positive control) induced a doses dependent decrease in cell viability while Ugambu and Product Blue did not affect cell viability in treated PBMCs. Ihashi, Product Nene and SDKc induced a dose dependent decrease in cell viability with high doses showing significant $(p<0.001)$ toxicity to treated PBMCs. SPNa doses were also significantly $(p<0.001)$ toxic to PBMCs even in low doses.

http://dx.doi.org/10.4314/ajtcam.v9i3S.1 


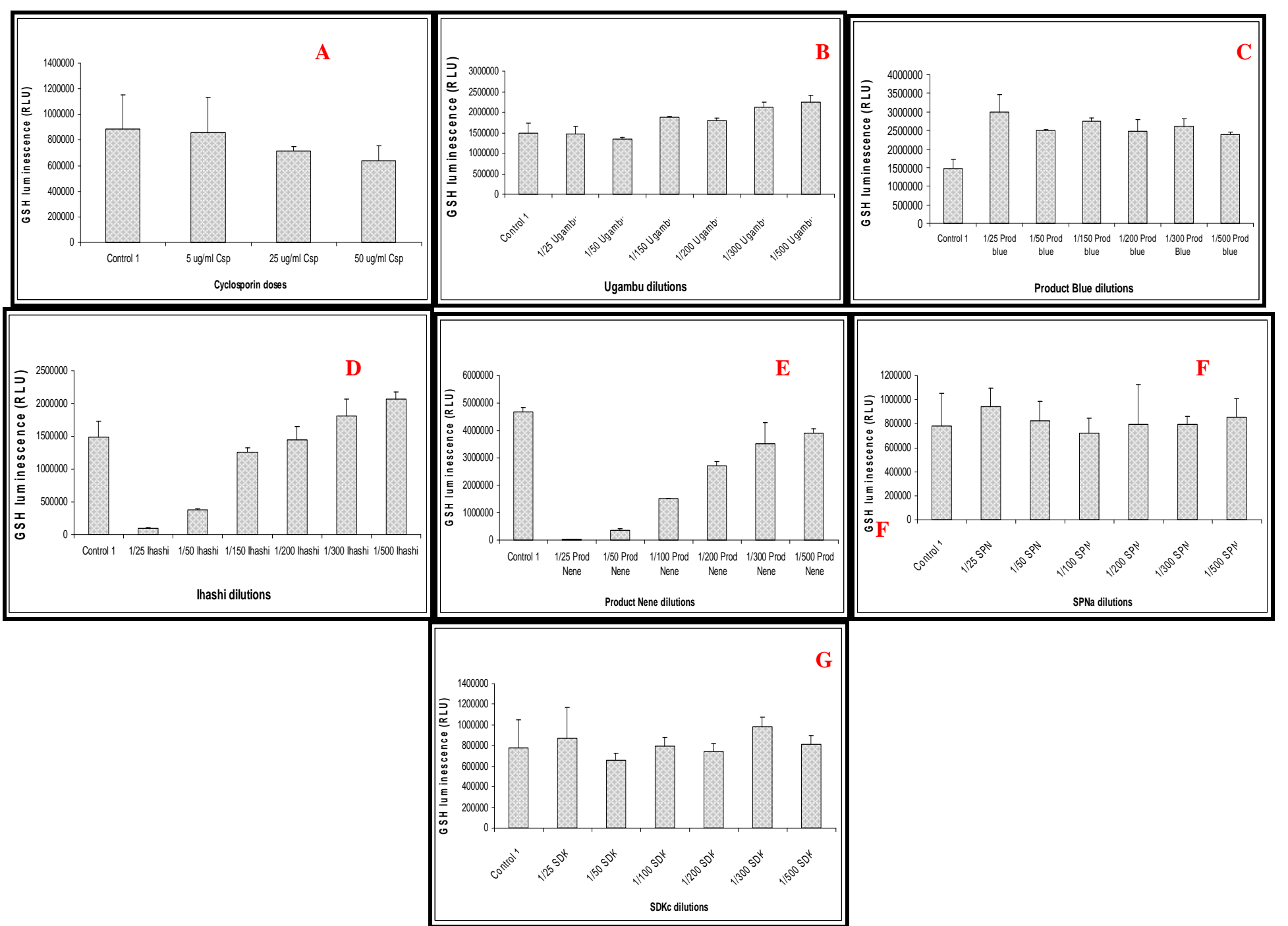

Figure 2: Changes in intracellular levels of glutathione (GSH) of treated and control samples of PBMCs after 24 hours of incubation. The dose response curves represented include (A) cyclosporine, (B) Ugambu, (C) Product Blue, (D) Ihashi, (E) Product Nene, (F) SPNa, and (G) SDKc. The positive control was cyclosporine and each graph included an untreated control as a reference sample. Ugambu high doses decreased GSH levels while low doses induced an increase in this intracellular antioxidant. Ihashi and Product Nene showed adose dependent effect on GSH levels with high doses significantly $(p<0.001)$ decreasing GSH content while low dose were tolerated by treated PBMCs. SPNa and SDKc did not change GSH content of treated PBMCs significantly from those of untreated cells. 
Table 2: Cytokine analyses results from supernatants of PBMCs treated with doses of Product Nene, SPNa and SDKc over 24 hours.

\begin{tabular}{|c|c|c|c|c|c|c|c|c|c|}
\hline & \multicolumn{4}{|c|}{ Product Nene } & \multicolumn{4}{c|}{ SPNa } & \multicolumn{3}{c|}{ SDKc } \\
\cline { 2 - 11 } Cytokines & \multicolumn{9}{|c|}{ Spectrophotometric absorbance readings } \\
\cline { 2 - 11 } & Control & $\mathbf{1 / 2 0 0}$ & $\mathbf{1 / 5 0 0}$ & Control & $\mathbf{1 / 2 0 0}$ & $\mathbf{1 / 5 0 0}$ & Control & $\mathbf{1 / 2 0 0}$ & $\mathbf{1 / 5 0 0}$ \\
\hline IL 1 $\alpha$ & 0.002527 & $0.145798^{*}$ & $0.175523^{*}$ & 0.010246 & 0.030728 & 0.039901 & 0.010246 & 0.021322 & $0.032847^{*}$ \\
\hline IL 1 $\beta$ & 0.077016 & $2.259161^{*}$ & $2.28086^{*}$ & 0.029211 & 0.061474 & 0.091266 & 0.029211 & $0.074271^{*}$ & $0.095577^{*}$ \\
\hline IL 2 & 0 & 0.008973 & 0.012604 & 0 & 0.014484 & 0.005585 & 0 & 0 & 0 \\
\hline IL 4 & 0.0206 & 0.03648 & 0.023376 & 0 & 0.050615 & 0.047132 & 0 & $0.04001^{*}$ & $0.048964^{*}$ \\
\hline IL 6 & 2.03003 & $2.314435^{*}$ & $2.306163^{*}$ & 0.200273 & $0.408549^{*}$ & 0.278515 & 0.200273 & $0.448786^{*}$ & 0.259028 \\
\hline IL 8 & 1.509134 & $1.843873^{*}$ & $2.221598^{*}$ & 1.229702 & 1.532653 & 1.550086 & 1.229702 & 1.45217 & 1.234844 \\
\hline IL 10 & 0.048767 & $0.978975^{*}$ & $0.627364^{*}$ & 0.006483 & $0.032181^{*}$ & $0.051847^{*}$ & 0.006483 & $0.07851^{*}$ & $0.079002^{*}$ \\
\hline IL 12 & 0.013905 & 0.034357 & 0.017545 & 0.042441 & 0.044058 & 0.055228 & 0.042441 & 0.037429 & 0.041418 \\
\hline IL 17 $\alpha$ & 0.00524 & 0.009919 & 0.012963 & 0.0307 & $0.117735^{*}$ & 0.050291 & 0.0307 & $0.072203^{*}$ & $0.058431^{*}$ \\
\hline IFN $\gamma$ & 0 & $0.031149^{*}$ & 0.016341 & 0.018318 & 0.028502 & 0.035927 & 0.018318 & $0.050088^{*}$ & $0.059476^{*}$ \\
\hline TNF $\alpha$ & 0 & $1.0211^{*}$ & $0.484274^{*}$ & 0.041442 & $0.082701^{*}$ & $0.067795^{*}$ & 0.041442 & $0.093146^{*}$ & $0.073614^{*}$ \\
\hline GM-CSF & 0.001021 & $0.255015^{*}$ & $0.250927^{*}$ & 0.049034 & 0.043058 & $0.0684^{*}$ & 0.049034 & $0.080871^{*}$ & $0.075226^{*}$ \\
\hline
\end{tabular}

*Significant change in absorbance readings for each cytokine as compared to untreated control sample

\section{XTT screening assay}

The screening assay measured the ability of the traditional medicinal preparations to protect MT-4 against HIV induced autolysis. Because the study was not designed to pinpoint the exact mechanism of action of each medicinal preparation, any antiretroval drug could be used as a positive control. Azidothymidine (AZT) was therefore chosen as a positive control. Ugambu, Ihashi, Product Nene, and SDKC showed the highest protective effect while SPNa and Product Blue had negligent or zero protective effect. These products had no significant protective effects and also had very low ability to selectively distinguish between infected and non-infected cells when compared to AZT ( $p>0.05)$ (Table 3).

Table 3: Results from the XTT screening assay

\begin{tabular}{|c|c|c|c|}
\hline $\begin{array}{c}\text { Traditional medicine } \\
\text { preparation }\end{array}$ & IC $_{\mathbf{5 0}}$ (dilutions) & $\mathbf{E C}_{\mathbf{5 0}}$ (dilutions) & SI \\
\hline AZT & 0.044 & $4.43 \times 10^{-5}$ & 1009 \\
\hline 1 Ugambu & -3.589 & -1.4 & 0.03 \\
\hline 2 SPNa & -2.178 & -2.6 & 2.02 \\
\hline 3 Ihashi & -1.9 & -3.5 & 13.1 \\
\hline 4 SDKc & -1.83 & -5.1 & 0.016 \\
\hline 5 Product Nene & -3.08 & -0.5 & - \\
\hline 6 Product Blue & -0.429 & - & \\
\hline
\end{tabular}

\section{HIV-1 Viral loads}

The purpose of the experiment was to assess antiviral activity and not mechanism(s) of action; hence any antiretroviral drug with known antiviral activity could be used, independent of its mechanism of action. According to the adopted innovative method based on the on the algorithm for HIV viral load testing, one test is sufficient. It is important to note that the assay was setup in a diagnostic level 3 laboratory. The antiviral activity of herbal medicines was tested on MT-4 cells incubated with the HIV-1 virus and treated with several dilutions of traditional medicine preparations and compared to the antiviral drug AZT. 
Ugambu, Ihashi, and Product Nene showed the highest antiviral activity. Over the five days of incubation, these products had higher antiviral activity than the positive control AZT (Figure 3). SDKC, SPNa and Product Blue did not show any significant antiviral properties when compared to AZT (Figure 3).

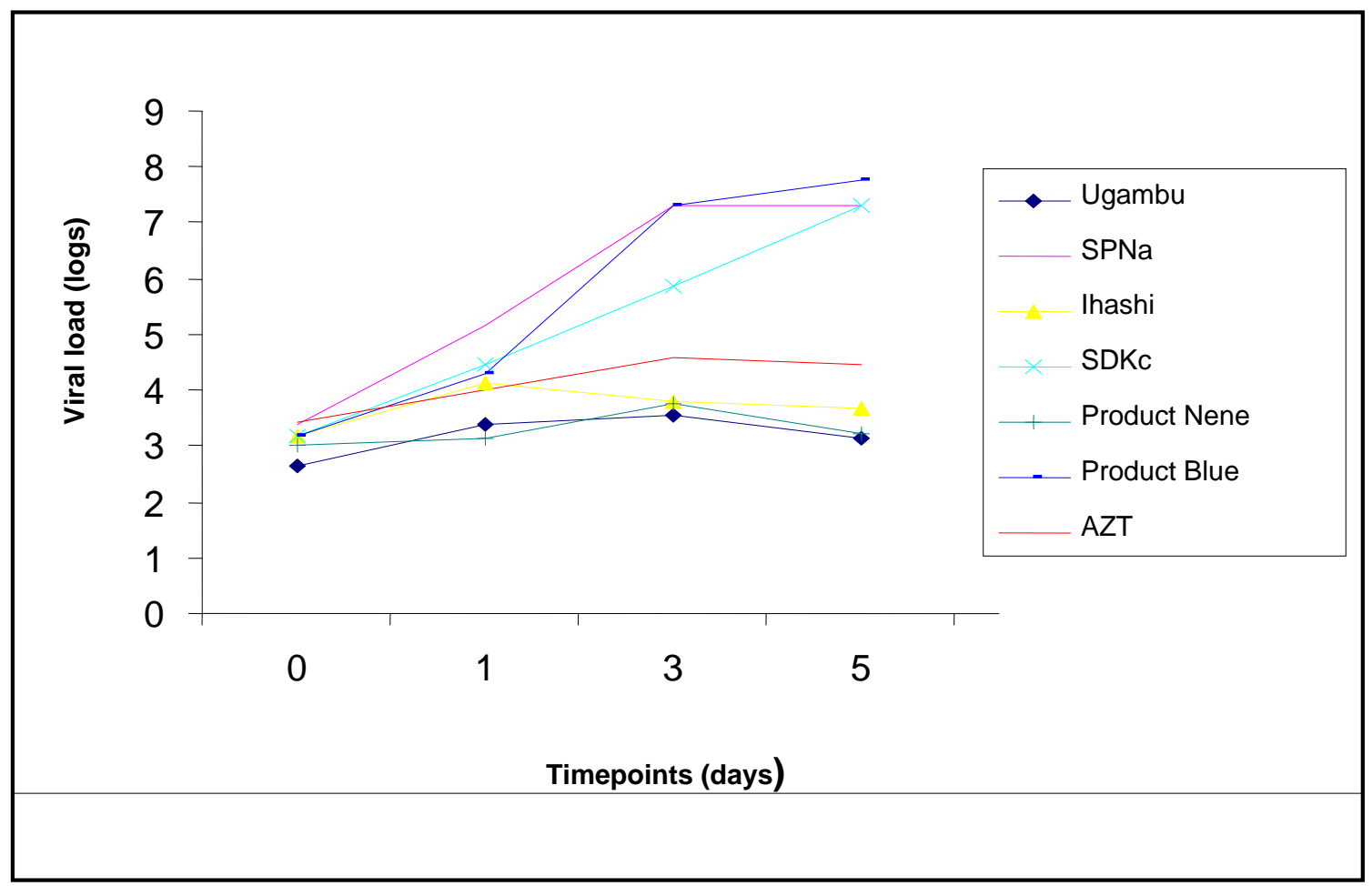

Figure 3: $\log _{10}$ HIV Viral loads of culture supernatant exposed to traditional medicine preparations 1 to 6 (as outlined in table 3) and AZT at $0,1,3$ and 5 days following infection of MT-4 cells by HTLV IIIB. The concentration of the plant extracts Ugambu, SPNa, Ihashi, SDKc, Product Nene and Product Blue was equivalent to that of the $\mathrm{EC}_{50}-1.4,-2.6,-3.5,-5.1$, and -0.5. AZT had an $\mathrm{EC}_{50}$ of $4.43 \times 10^{-5}$.

\section{Discussion}

Many plants used in traditional medicine preparations have immunostimulant and immunomodulatory properties. Such effects are important in altering the human immune response to fight infections such as HIV and AIDS (Bodeker et al., 2006). The purpose of this study was to evaluate properties of multiple component traditional medicine preparations prescribed by local THPs to patients with compromised immune systems. Traditional medicine preparations such Ugambu, Ihashi, Product Nene and SDKc showed a dose dependent effect in the levels of ATP and GSH in treated immune cells. High doses of these products were cytotoxic to PBMCs while high dilutions did not deplete either ATP or GSH. Such dose dependent responses are normal for most drugs as shown by the positive control, cyclosporine. Depletion of ATP and GSH levels also determine the type of cell death induced in treated cells. The same reagent might induce apoptosis at lower dose but induce cell death by necrosis at higher doses. This is probably due to the collapse of cellular integrity, including plasma membrane disruption, a large calcium influx, as well as intracellular ATP depletion (Tsujimoto, 1997).

No significant cytoxicity was detected in PBMCs treated with dilutions of Product Blue. This product enhanced the levels of ATP and GSH content of all treated samples. While Product Blue may seem to enhance the ability of immune cells to resist infections, it did not modulate the production of cytokines at medium to low doses. Medicinal preparations Ugambu, Ihashi, Product Nene, SPNa and SDKc, which showed relative cytoxicity at high doses, were able to modulate pro-inflammatory cytokines at non-cytotoxic low doses. Immune cells treated with these products demonstrated increased secretion of IL $1 \beta$, IL 2 , IL 8 , IFN- $\gamma$, TNF- $\alpha$, and GM-CSF $(p<0.001)$. Such inflammatory cytokines are multi-potential mediators of the cellular immune system with a wide variety of biological activities. Traditional herbal medicines which can stimulate the immune system through inducing cytokine production in healthy PBMCs may also have positive effects on HIV-infected patients (Barak et al., 2002; Liang et al., 2008). It is important to also note that the treated PBMCs in this study were not stimulated and therefore expected to 
secrete less cytokines. A study by Hendricks and Pool (2010) on the effects of Rooibos tea on immune cells showed that cytokines are expressed at very low concentrations in unstimulated immune cells while stimulation with endotoxin lipopolysaccharide (LPS) dramatically increase cytokine expression.

Traditional medicine preparations such as Ugambu, Ihashi, and Product Nene, which showed an ability to significantly modulate cytokine secretion in normal PBMCs, also had the highest antiviral activities of all the products tested. It is therefore possible that antiviral properties of these products is related to their ability modulate cytokine secretion in treated immune cells even though this study does not prove it. Multi-purpose traditional herbal preparations sold in South Africa showed potent activity against HIV reverse transcriptase (RT) enzyme (Ndhlala et al., 2010). It is important to note that the herbal preparations which showed high antiviral activities were also cytotoxic to normal immune cells. The same medicinal preparations had very low selective indexes in the XTT screening assay. Therefore finding an effective in vitro dose which does not affect viability of noninfected cells requires a fine balance.

\section{Conclusion}

The data obtained from in vitro assays in this study has shown that some of the traditional medicines have at least one or all the properties of immunostimulation, immunomodulation or antiretroviral effects. Specific studies must be conducted to ascertain each mechanism of action of these multi herbal preparations because the data generated in this study suggest multiple sites of activity. Active metabolites involved in the observed biological effects must also be isolated. This work should directly involve the knowledge holders of the traditional medicines.

\section{Acknowledgments}

Funding for this research was provided by the National Research Foundation through the South African Chair in Indigenous Healthcare Systems, Professor Nceba Gqaleni. The Traditional Health Practitioners who voluntarily brought their medicinal mixtures to be studied are also acknowledged. Some of the reagents for the screening and viral load assay were obtained through the NIH AIDS Research and Reference Reagent Program, Division of AIDS, NIAID, NIH: H9/HTLV-IIIB NIH 1983 from Dr. Robert Gallo and MT-4 from Dr. Douglas Richman

\section{References}

1. Barak, V., Birkenfeld, S., Halperin, T., Kalickman, I. (2002). The effect of herbal remedies on the production of human inflammatory and anti-inflammatory cytokines. IMAJ 4: 919-922.

2. $\quad$ Bessong, P.O., Obi C.L., Igumbor, E., Andreola, M.L., Litvak, S. (2004). In vitro activity of three selected South African plants against human immunodeficiency virus type 1 reverse transcriptase. Afr. J. Biotechnol. 3: 555-559.

3. Bodeker, G., Carter, G., Burford, G., Dvorak-Little, M. (2006). HIV/AIDS: Traditional systems of health care in the management of a global epidemic. J. Altern. Compl. Med. 12(6): 563-576.

4. Chen, X., Howard, O.M.Z., Yang, X., et al. (2002). Effects of Shuanghuanglian and Qingkialing, two multi-components of traditional Chinese medicinal preparations, on human leukocyte function. Life Sci. 70: 2897-2913.

5. Cowan, M.M. (1999). Plant products as antimicrobial agents. Clin. Micro. Rev. 12(4): 564-582.

6. Haddad, P.S., Georges, A.A., Groom, S., Boivin, M. (2005). Natural health products, modulation of immune function and prevention of chronic diseases. eCAM 2(4): 513-520.

7. Hendricks, R., Pool, E.J. (2010). The in vitro effects of Rooibos and Black tea on immune pathways. J. Immunoassay Immunochem. 31: 169-180.

8. Liang, H., Loo, W.T.Y., Yeung, B.H.S., Cheung, M.N.B., Wang, M., Chen, J.P. (2008). A non-toxic herbal remedy which enhance lymphocytes activity and cytokine secretion: Ganoderma lucidum. Afr. J. Biotechnol. 7(22): 4010-4014.

9. $\quad$ Lowe, S.W., Lin, A.W. (2000). Apoptosis in cancer. Carcinog. 21: 485-495.

10. Ndhlala, A.R., G.I., Finnie, J.F., Van Staden, J. (2010). In vitro antioxidant properties, HIV-1 reverse transcriptase and acetylcholinesterase inhibitory effects of traditional herbal preparations sold in South Africa. Molecules 15: 6888-6904.

11. Ndhlala, A.R., Stafford, G.I., Finnie, J.F., Van Staden, J. (2009). In vitro pharmacological effects of manufactured herbal concoctions used in KwaZulu-Natal South Africa. J. Ethnopharmacol.122: 117-122.

12. Nijnik, A., Madera, L., Ma, S., et al. (2010). Synthetic cationic peptide IDR-1002 provides protection against bacterial infections through chemokine induction and enhanced leukocyte recruitment. J. Immunol. 184: 2539-2550.

13. Roehm, N.W., Rodgers, G.H., Hatfield, S.M., Glasebrook, A.L. (1991). An improved colorimetric assay for cell proliferation and viability utilizing the tetrazolium salt XTT. J. Immunol. Methods 142: 257-265.

14. Scudiero, D.A., Shoemaker, R.H., Paull, K.D., Monks, A., Tieney, S., Hafziget, T.H., Currens, J.J., Sheriff, D., Boyd, M.R. (1988). Evaluation of soluble tetrazolium/formazan assay for cell growth and drug sensitivity in culture using human and other tumor cell lines. Cancer Res. 48:4827-4833. 
15. Spelman, K.M.S., Burns, J.J.N.D., Nichols, D.N.D., et al. (2006). Modulation of cytokine expression by traditional medicines: a review of herbal immunomodulators. Altern. Med. Rev. 11(2): 128- 150.

16. Tshibangu, K.C., Worku, Z.B., De Jongh, M.A., van Wyk, A.E., Mokwena, S.O., Peronovic, V. (2004). Assessment of effectiveness of traditional medicine in managing HIV/AIDS patients in South Africa. East Afr. Med. J. 81(10): 499-504.

17. Tsujimoto, Y. (1997). Apoptosis and necrosis: intracellular ATP levels as a determinant for cell death modes (review). Cell Death Differ. 4: 429-434.

18. Weislow, O.S., Kiser, R., Fine, D.L., Bader, J., Shoemaker, R.H., Boyd, M.R. (1989). New soluble-formazan assay for HIV-1 cytopathic effects: Application to high-flux screening of synthetic and natural products for AIDS-antiviral activity, J. Natl Cancer Inst. 81(8):577-586.

19. World Health Organization, (2005). National policy on traditional medicine and regulation of herbal medicines: Report of a WHO global survey. 